\title{
Effects of Peer Education Intervention for Hepatitis B on Level of Knowledge and Beliefs of School Adolescents in Jos, Plateau State Nigeria
}

\author{
Mary Mathew ${ }^{1 *}$, Femi Rufus Tinuola ${ }^{2}$, Sonika Raj Goel ${ }^{3}$, Olaniyi Taiwo ${ }^{4}$ and Jalo Philip ${ }^{4}$ \\ ${ }^{1}$ Abnira Medical Centre, Jos, Nigeria \\ ${ }^{2}$ Adekunle Ajasin University, Akoko, Ondo State, Nigeria \\ ${ }^{3}$ Texila American University, Nicaragua, Nigeria \\ ${ }^{4}$ Department of Basic Sciences and Research, ICOH for Africa, Jos, Nigeria \\ *Corresponding Author: Mary Mathew, Abnira Medical Centre, Jos, Nigeria.
}

Received: September 27, 2019; Published: October 10, 2019

DOI: $10.31080 /$ ASNH.2019.03.0486

\section{Abstract}

A high average prevalence rate of $11-13.7 \%$ for viral hepatitis B exists in Nigeria. This study examined the impact of a peer education intervention among senior secondary school students on hepatitis B knowledge and beliefs in six public day secondary schools located in Jos South Local Government Area, Plateau state.

A quasi-experimental study was carried out on six hundred students selected by a multistage sampling technique. A pre-tested formal self-administered questionnaire (SAQ) was used to collect data pre and twelve weeks post intervention. Chi- square and students t-test was done to compare intervention and control groups on outcome variables. Results showed the age distribution of the respondents was 1024 years with majority of respondents (86.7\%) in the age group 15-19 years. The independent sample test showed there was a significant difference in the mean for level of knowledge before the peer education intervention $(\mathrm{M}=9.07, \mathrm{SD}=$ 5.68) and after the peer education $(M=14.8, S D=4.8)$ intervention; $p=0.001$ suggesting that peer education really does have an effect on the level of knowledge for HBV. In addition, an independent samples t-test conducted to compare level of knowledge with peer education intervention and no peer education condition showed a significant difference in the scores for peer education intervention $(M=14.8, S D=4.8)$ and no peer education $(M=13.8, S D=4.3)$ conditions; $p=0.005$. Regarding beliefs, a significant difference in the use of condoms $(\chi 2=48.19 ; \mathrm{p}=0.001)$ post intervention between intervention and control group using chi-square test was found.

Keywords: Peer Education; Pre Intervention; Post Intervention; Hepatitis B; Control Group; Secondary School Student

\section{Introduction}

Nigeria contributes significantly to the burden of chronic viral hepatitis globally. Viral hepatitis is the seventh leading cause of death globally [1]. An estimated 95\% of individuals with chronic HBV or HCV infection, or both, are unaware of their infection and so do not benefit from clinical care, treatment, and interventions that are designed to reduce onward transmission [2]. Majority of the Nigerian population are not aware of its chronic complications of liver cirrhosis and primary liver cell cancers [3]. Lack of awareness of HBV, its risk factors, and its consequences are recognized as major deterrents to adopting positive preventive behavior including immunization among HBV high-risk groups [4].

The risk of Hepatitis B Virus (HBV) infection being chronic is inversely related to the age of infection and so there is a risk of $20-50 \%$ after childhood infection (aged $<5$ years) but less than $5 \%$ for adults infected after age 20 years [2]. With hepatitis B infec- tion as a significant opportunist infection among people living with Human Immunodeficiency Virus (HIV) [5], it is more worrisome as the risk of transmitting these infections to other members of the communities is high as they share common modes of transmission.

The study among senior school (SS) students (16-25 years of age) in public day schools contributed towards not only getting evidence about the level of knowledge and beliefs among the young population but their willingness to go for a hepatitis B test and use of condoms for sexual relationships before and after a peer education intervention. Apart from clinical research on viral hepatitis, publications on public health issues focusing on hepatitis and peer education in Nigeria are sparse. This research therefore was the first study carried out in Jos South Local Government Area, Plateau state, Nigeria to assess knowledge and beliefs of senior secondary students before and after a peer education intervention. 


\section{Methods}

A Quasi-experimental study peer education intervention among SS3 students in Jos public day Senior Secondary Schools, Jos South Local Government Area, Plateau state, Nigeria to assess knowledge and beliefs about hepatitis B was done in three stages: pre- intervention, intervention, post-intervention stage. Eligibility criteria consisted of inclusion criteria: a student aged between 1025 years, male or female, living with either one or both parents, and voluntarily participation in the study. Exclusion criteria were students below the age of ten and twenty-six or above, living with someone else apart from parents, and failure to agree voluntarily to participate in the study.

The sample was drawn using a multistage sampling technique. Day Senior Secondary schools in Jos South Local Government Area, Jos constituted the sampling frame. The primary sampling units was Day Senior Secondary schools (3 Day Senior Secondary schools as intervention schools and 3 Day Senior Secondary schools as controls) selected by random sampling. The 'control' schools selected were matched with 'intervention' schools based on same location so that one ensured the respondents in control and intervention schools shared similar characteristics. The secondary sampling units were two streams of Senior Secondary class in the selected 6 secondary schools (as the Day Senior Secondary schools have an average of three streams per Senior Secondary class) while the tertiary sampling units were the individual students in the class, selecting 50 students in each class/stream. The selection of 50 students in each class/stream (two classes/school) for the six schools by simple random sampling method made a total number of 600 respondents.

For comparing two proportions, the sample size formula used was $n=(Z \alpha / 2+Z \beta) 2 *(p 1(1 p 1)+p 2(1-p 2)) /(p 1-p 2) 2$, where $n$ is the sample size for a proportion, $Z \alpha / 2$ is the critical value of the Normal distribution at $\alpha / 2$ (for a confidence level of $95 \%, \alpha$ is 0.05 and the critical value is 1.96), $Z \beta$ is the critical value of the Normal distribution at $\beta$ (for a power of $80 \%, \beta$ is 0.2 and the critical value is 0.84 ) and $\mathrm{p} 1$ and $\mathrm{p} 2$ are the expected sample proportions of the two groups. For detecting a difference between two proportions, $\mathrm{p} 1$ was taken as 0.3 and $\mathrm{p} 2$ as 0.2 [6]. Imputing values, $\mathrm{n}=(1.96+$ $0.84) 2 *(0.3(1-0.3)+0.2(1-0.2)) /(0.3-0.2) 2 n=290.08$.

Considering a probable non-response of $3 \%$ with $n=300$ for each proportion, the sample size amounted to a total of 600 . Research study was done in three stages: pre- intervention, intervention, postintervention stage.

\section{Pre - intervention}

This was done by the administration of formal self-administered questionnaire (SAQ) containing open and close- ended questions, both before and after the peer education intervention to both interventions and controls Day Senior Secondary schools though the peer education intervention was only conducted in the three 'intervention' Day Senior Secondary schools. The questionnaires administered before the peer education intervention served as the baseline assessment of knowledge and beliefs.

\section{Intervention}

Fifteen students ( 5 per intervention school for 3 schools) were selected as 'Peer Educators' (PEs) using the following criteria: student in the select Day Senior Secondary School aged between 1025 years, good character, recommended by the school authority as being intelligent, good oral communication skills, active listening skills and able to keep confidentiality. A supervisor of the PEs, preferably a class or subject teacher was also chosen for each school. Both the PEs and teacher supervisor were trained by me with the aim of giving them knowledge on hepatitis and increasing their skills for effective communication and behavior change.

A single peer education intervention of thirty minutes was conducted in each 'intervention' school a week after the baseline assessment by the trained PEs consisting of knowledge on basic facts of hepatitis B including mode of transmission, prevention and control measures for hepatitis B and its association with Human Immunodeficiency Virus/Acquired Immunodeficiency Syndrome and hepatitis C. One-on-one peer sessions were also held on student request during break time.

\section{Post - intervention}

Post intervention data was collected twelve weeks later in all schools (intervention and control). Free voluntary hepatitis B tests was offered to those students who want to undertake the hepatitis B surface antigen blood test in intervention schools.

Blood samples were obtained by registered laboratory scientists for qualitative detection of HBsAg using rapid chromatographic immunoassays with test kits from ABON (China) having sensitivity, specificity and accuracy of $>99 \%, 97 \%$ and $98.5 \%$ respectively. Universal precautions and standard protocols for hepatitis B testing were adhered to.

Data analysis was done in stages. The collected data questionnaires were 'cleaned'. Data was then entered into the computer using Microsoft excel, which was then exported for analysis with the aid of the Statistical Package for Social Sciences (SPSS) 22.0 computer statistical software. Exploratory analysis to check for missing fields was then done. Descriptive statistics summarizing numerical data of age, categorical data of sex and tribe was displayed by frequency tables and graphical representation of observations. This was followed by data analysis done keeping the study objectives and hypothesis in mind. Statistical tests such as students t-test and chi-squared was done to compare intervention and control groups on outcome variables. Results of chi-square values obtained from 
the study were considered significant at $\mathrm{p} \leq 0.05$. Knowledge/ belief levels data was converted from qualitative (categorical) to quantitative (continuous) data for analysis using a total of 26 score. Grading of score was done and the Likert scale was: Poor (0-9); Average (10-19) and Good (20-26).

Ethical approval process involved written approval from the Plateau state government, Local Government, parents/respondents and the ethical committee (state government approved). Participants were asked to give written consent at the beginning of the questionnaire regarding acceptance of voluntary responses and informed written consent got to do the hepatitis B test. For students below the age of 18 years, informed consent for hepatitis B testing was obtained from the student's parents.

\section{Results}

Six hundred respondents were involved in the study of which 241 were males, 355 were females and 4 non-responses. 300 respondents each in control and intervention study participated. Significant findings were found twelve weeks post intervention at end of study.

\section{Discussion}

The age distribution of the respondents was 10-24 years with a mean age of $16.65 \pm 1.72 \mathrm{yrs}$. Age in both groups, control and inter- vention showed similar age characteristics (Table 1). Majority of respondents (86.7\%) were in the age group 15-19 years (Figure 1). With infection common among 20-40 years [7], this is the best age to focus on to prevent HBV by increasing knowledge and awareness to HBV and changing beliefs/attitudes and cultural norms that facilitate transmission of the disease. More so, as awareness and risk perception on HBV infection are high in Jos among tertiary education institution students (University of Jos), but uptake of HB vaccine low and findings worst for non-health students [8].

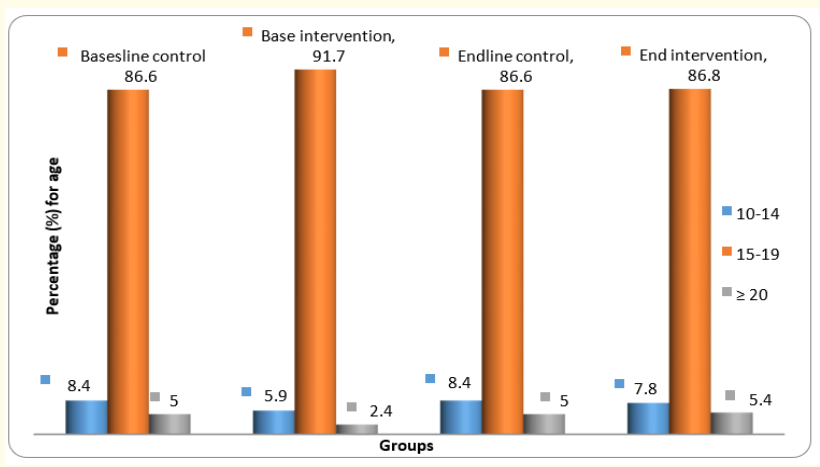

Figure 1: Bar chart showing age distribution between control and intervention groups before and after a peer education intervention on hepatitis B.

\begin{tabular}{|l|c|c|c|c|c|c|}
\hline \multicolumn{1}{|c|}{ Age } & \multicolumn{2}{c|}{ Baseline } & Total & \multicolumn{2}{c|}{ End } & Total \\
\hline & Control & Intervention & & Control & Intervention & \\
\hline $10-14$ & $25(8.4)$ & $17(5.9)$ & $42(7.2)$ & $25(8.4)$ & $23(7.8)$ & $48(8.1)$ \\
\hline $15-19$ & $258(86.6)$ & $265(91.7)$ & $523(89.1)$ & $258(86.6)$ & $256(86.8)$ & $514(86.7)$ \\
\hline$\geq 20$ & $15(5.0)$ & $7(2.4)$ & $22(3.7)$ & $15(5.0)$ & $16(5.4)$ & $31(5.2)$ \\
\hline TOTAL & $298(100.0)$ & $289(100.0)$ & $587(100.0)$ & $298(100.0)$ & $295(100.0)$ & $593(100.0)$ \\
\hline
\end{tabular}

Table 1: Distribution of study participants according to age at baseline and end of study.

Table 2 shows that female participants were more than males at both baseline and at end. The table also shows that majority of respondents were from other tribes (7.5.3\%) with respect to ethnicity, mainly locally based and indigenes of Plateau state. This indicates that findings from the study will truly reflect the socioeconomic norms of the people of the state. Majority of respondents were Christians staying with their parents (Table 3). Interventions targeting parents also needs to be recommended and included in strategizing to prevent disease transmission. Tung and Middleman [9] evaluated school level factors in a successful school-based HBV immunization program and found parent involvement resulted in enrollment and return of consent/refusal form phases of immunization initiatives and maximise number of school children immunised.

Tables 4, 5 and 6 tested the first null hypothesis (There is no significant difference in level of knowledge about hepatitis $B$ among students in intervention and control group) in line with achieving the first objective of the research study. The results from the three tables collectively indicated significant difference in the level of knowledge between control and intervention groups before and after the peer education intervention.

Results for level of knowledge both before and after intervention are reported in Table 4. The independent sample t-test showed there was a significant difference in the mean for level of knowledge before the peer education intervention $(M=9.07, S D=5.68)$ and after the peer education $(\mathrm{M}=14.8, \mathrm{SD}=4.8)$ intervention; $\mathrm{p}=$ 0.001 . These results suggest that peer education really does have an effect on the level of knowledge for HBV.

An independent samples t-test (Table 5) was conducted to compare level of knowledge with peer education intervention and no peer education condition. There was a significant difference in the scores for peer education intervention $(M=14.8, S D=4.8)$ and no peer education $(M=13.8, S D=4.3)$ conditions; $p=0.005$. These results suggest that peer education really does have an effect on the level of knowledge for HBV. Specifically, our results suggest that 
Effects of Peer Education Intervention for Hepatitis B on Level of Knowledge and Beliefs of School Adolescents in Jos, Plateau State Nigeria

\begin{tabular}{|l|c|c|c|c|c|c|}
\hline & \multicolumn{2}{|c|}{ Baseline } & TOTAL & \multicolumn{2}{c|}{ End } & TOTAL \\
\hline Sex & Control & Intervention & & Control & Intervention & \\
\hline Male & $145(48.5)$ & $123(41.4)$ & $268(45.0)$ & $145(48.5)$ & $96(32.3)$ & $241(40.4)$ \\
\hline Female & $154(51.5)$ & $174(58.6)$ & $328(55.5)$ & $154(51.5)$ & $201(67.7)$ & $355(59.6)$ \\
\hline TOTAL & $299(100.0)$ & $297(100.0)$ & $596(100.0)$ & $299(100.0)$ & $297(100.0)$ & $596(100.0)$ \\
\hline Ethnicity & Control & Intervention & & Control & Intervention & \\
\hline Hausa & $31(10.5)$ & $50(17.4)$ & $81(13.9)$ & $31(10.5)$ & $45(16.9)$ & $76(13.7)$ \\
\hline Igbo & $23(7.8)$ & $33(11.5)$ & $56(9.6)$ & $23(7.8)$ & $13(4.9)$ & $36(6.3)$ \\
\hline Yoruba & $11(3.7)$ & $12(4.2)$ & $23(3.9)$ & $11(3.7)$ & $15(5.6)$ & $26(4.7)$ \\
\hline Others & $231(78.0)$ & $192(66.9)$ & $423(72.6)$ & $231(78.0)$ & $193(72.6)$ & $424(75.3)$ \\
\hline TOTAL & $296(100.0)$ & $287(100.0)$ & $583(100.0)$ & $296(100.0)$ & $266(100.0)$ & $596(100.0)$ \\
\hline
\end{tabular}

Table 2: Distribution of study participants according to sex and ethnicity at baseline and end of study.

\begin{tabular}{|l|c|c|c|c|c|c|}
\hline & \multicolumn{2}{|c|}{ Baseline } & TOTAL & \multicolumn{2}{c|}{ End } & TOTAL \\
\hline Religion & Control & Intervention & & Control & Intervention & \\
\hline Christianity & $285(96.9)$ & $257(88.9)$ & $542(93.0)$ & $286(97.3)$ & $253(91.0)$ & $539(94.2)$ \\
\hline Islam & $7(2.4)$ & $32(11.1)$ & $39(6.7)$ & $8(2.7)$ & $25(9.0)$ & $33(5.8)$ \\
\hline Others & $2(0.7)$ & $0(0.0)$ & $2(0.3)$ & $0(0.0)$ & $0(0.0)$ & $0(0.0)$ \\
\hline TOTAL & $294(100.0)$ & $289(100.0)$ & $583(100.0)$ & $294(100.0)$ & $278(100.0)$ & $572(100.0)$ \\
\hline Currently living with parents & & & & & & \\
\hline Yes & $208(72.5)$ & $185(65.8)$ & $393(69.2)$ & $208(72.5)$ & $198(71.0)$ & $406(71.8)$ \\
\hline No & $79(27.5)$ & $96(34.2)$ & $175(30.8)$ & $79(27.5)$ & $81(29.0)$ & $160(28.2)$ \\
\hline TOTAL & $287(100.0)$ & $281(100.0)$ & $568(100.0)$ & $287(100.0)$ & $279(100.0)$ & $566(100.0)$ \\
\hline
\end{tabular}

Table 3: Distribution of respondents according to religion and parents at baseline and end of study.

\begin{tabular}{|l|c|c|c|}
\hline \multicolumn{1}{|c|}{ Period } & Mean \pm SD knowledge score & t-test & P-value \\
\hline Raw scores & & & \\
Before intervention & $9.07 \pm 5.68$ & 13.113 & \\
\hline After intervention & $14.81 \pm 4.80$ & & \\
\hline Percentage score & & 13.113 & 0.001 \\
Before intervention & $34.87 \pm 22.61$ & & \\
\hline After intervention & $56.97 \pm 18.46$ & & \\
\hline
\end{tabular}

Table 4: Assessment of level of knowledge of senior secondary school students before and after a peer education intervention about hepatitis B.

\begin{tabular}{|l|c|c|c|}
\hline \multicolumn{1}{|c|}{ Group } & Mean \pm SD knowledge score & t-test & P-value \\
\hline Raw scores & & & \\
Control group & $13.77 \pm 4.26$ & 2.824 & 0.005 \\
\hline Intervention group & $14.81 \pm 4.80$ & & \\
\hline Percentage score & & 2.824 & 0.005 \\
Control group & $52.95 \pm 16.40$ & & \\
\hline Intervention group & $56.97 \pm 18.46$ & & \\
\hline
\end{tabular}

Table 5: Assessment of level of knowledge of senior secondary school students in control and intervention groups of a peer education intervention about hepatitis B. 


\begin{tabular}{|l|c|c|c|c|}
\hline Knowledge status & Period & & $\boldsymbol{\chi}^{2}$ & P-value \\
\hline & Before intervention & After intervention & & \\
\hline Poor & $162(54.0)$ & $43(14.3)$ & 117.010 & 0.001 \\
\hline Average & $131(43.7)$ & $210(70.0)$ & & \\
\hline Good & $7(2.3)$ & $47(15.7)$ & & \\
\hline Total & $300(100.0)$ & $300(100.0)$ & & \\
\hline
\end{tabular}

Table 6: Assessment of level of knowledge of senior secondary school students before and after a peer education intervention about hepatitis B.

when there is a peer education intervention among school adolescents, their level of knowledge increases.

A chi-square test was performed (Table 6) and a significant relationship found between levels of knowledge before and after the peer education, $\chi 2=117.01, p=0.001$. Value of $p$ below 0.05 was considered as statistically significant. We can be $95 \%$ confident that the relationship between the two variables is not due to chance.

Similar findings have been found in Ethiopia, using peer education intervention on HIV/AIDS knowledge and related sexual behaviors of secondary school students [10]. When the pre and post intervention data of each group were compared, comprehensive knowledge of HIV (P-Values $=0.004$ ) and willingness to go for HIV counseling and testing $(\mathrm{P}$-value $=0.01)$ showed significant differences among intervention group students during post intervention period. Another study done in Ibadan in 1999 compared the knowledge, attitude, and sexual risk behaviors of 233 senior students who received comprehensive health education intervention with 217 controls. Six months' post intervention revealed that the knowledge about HIV transmission and prevention was significantly higher (P 0.05) in the intervention group. Furthermore, 92.8\% of the intervention students as compared to only $56.7 \%$ of the controls felt AIDS constituted a problem in Nigeria, indicating better attitudes among the intervention group. The end line data also showed that there was a decrease in the number of sexual partners among the intervention students from 1.51 to 1.06, while their condom use increase. In conclusion, the education program was successful in improving the student's sexual practices as well as their knowledge and attitudes regarding HIV/AIDS (Fawole, Asuzu and Brieger, 1999).

Majority of respondents had heard of HBV (both control and intervention groups), commonly from 'media' which includes the internet. Parents as a group came second as source of information on HBV. Interestingly, as a side finding (Table 7), the study found teachers as a source of information was low before the intervention but significantly increased after the intervention. This means teachers in school need to have an intervention so that their knowledge, awareness improved and subsequently play an important role in ensuring students increase their knowledge about HBV.

\begin{tabular}{|l|c|c|c|c|c|c|c|c|}
\hline \multicolumn{2}{|c|}{ Baseline } & \multicolumn{3}{c|}{} & \multicolumn{2}{c|}{ End } & \\
\hline Source & Control & Intervention & $\chi^{2}$ & P value & Control & Intervention & $\chi^{2}$ & P value \\
\hline Parents & & & & & & & \\
\hline No & $90(32.0)$ & $93(39.4)$ & 3.054 & 0.081 & $90(32.6)$ & $98(36.0)$ & 0.711 & 0.399 \\
\hline Friends & $191(68.0)$ & $143(60.6)$ & & & $186(67.4)$ & $174(64.0)$ & & \\
Yes & $35(12.5)$ & $33(14.0)$ & 0.262 & 0.609 & $40(14.5)$ & $42(15.4)$ & 0.097 & 0.756 \\
\hline No & $246(87.5)$ & $203(86.0)$ & & & $236(85.5)$ & $230(84.6)$ & & \\
\hline Media & & & & & & & & \\
Yes & $137(48.8)$ & $86(36.4)$ & 7.929 & 0.005 & $137(49.6)$ & $128(47.1)$ & 0.365 & 0.546 \\
\hline No & $144(51.2)$ & $150(63.6)$ & & & $139(50.4)$ & $144(52.9)$ & & \\
\hline Teacher & & & & & & & & \\
Yes & $50(17.8)$ & $67(28.4)$ & 8.226 & 0.004 & $52(18.8)$ & $105(38.6)$ & 26.173 & 0.001 \\
\hline No & $231(82.2)$ & $169(71.6)$ & & & $224(81.2)$ & $167(61.4)$ & & \\
\hline $\begin{array}{l}\text { Reading } \\
\text { books Yes }\end{array}$ & $29(10.3)$ & $32(13.6)$ & 1.293 & 0.255 & $32(11.6)$ & $54(19.9)$ & 6.967 & 0.008 \\
\hline No & $252(89.7)$ & $204(86.4)$ & & & $243(88.4)$ & $218(80.1)$ & & \\
\hline
\end{tabular}

Table 7: Distribution of respondents according to 'Source of Information' at baseline and end of study. 
To assess the second objective of the research study (to identify beliefs regarding personal risk perception, abstinence and safe sex practices among senior secondary students before and after a peer education intervention about Hepatitis B in Jos, Plateau state, Nigeria), the above analysis was done with tables 8 to 10 . Table 8 regarding personal risk perception showed numbers that had not heard of HBV vaccination and had not done a hepatitis B test though it was not statistically significant. There was an interest to have a hepatitis B test as 'parents were keen to agree' as shown by Table 8 and the chi-square test performed. A significant relationship found between control and intervention groups after the peer education intervention, $\chi 2=4.48, p=0.001$.

\begin{tabular}{|c|c|c|c|c|c|}
\hline Beliefs & Control & Intervention & Total & $\chi^{2}$ & P-value \\
\hline \multicolumn{3}{|c|}{ Have you had vaccination against Hepatitis B? } & \multirow[b]{2}{*}{$181(31.8)$} & \multirow[b]{2}{*}{1.078} & \multirow[b]{2}{*}{0.299} \\
\hline Yes & $95(33.8)$ & $86(29.8)$ & & & \\
\hline No & $186(66.2)$ & $203(70.2)$ & $389(68.2)$ & & \\
\hline Total & $281(100.0)$ & $289(100.0)$ & $570(100.0)$ & & \\
\hline \multicolumn{6}{|c|}{ Have you done a Hepatitis B test? } \\
\hline Yes & $68(23.5)$ & $51(17.6)$ & $119(20.6)$ & 3.058 & 0.080 \\
\hline No & $221(76.5)$ & $238(82.4)$ & $459(79.4)$ & & \\
\hline Total & $289(100.0)$ & $289(100.0)$ & $578(100.0)$ & & \\
\hline \multicolumn{6}{|c|}{ Do you think your parents will agree to you having Hepatitis test? } \\
\hline Yes & $219(80.2)$ & $245(86.9)$ & $464(83.6)$ & & \\
\hline No & $54(19.8)$ & $37(13.1)$ & $91(16.4)$ & 4.488 & 0.001 \\
\hline Total & $273(100.0)$ & $282(100.0)$ & $555(100.0)$ & & \\
\hline
\end{tabular}

Table 8: Distribution of respondents on beliefs (post -intervention) regarding personal risk perception of hepatitis B among group of senior secondary students in Jos Nigeria.

\begin{tabular}{|c|c|c|c|c|c|}
\hline Safe sex practices & Control & Intervention & Total & $\chi^{2}$ & P-value \\
\hline \multicolumn{6}{|l|}{ Have you ever had sex? } \\
\hline Yes & $86(30.2)$ & $51(17.1)$ & $137(23.5)$ & 13.825 & 0.001 \\
\hline No & $199(69.8)$ & $247(82.9)$ & $446(76.5)$ & & \\
\hline Total & $285(100.0)$ & $298(100.0)$ & $583(100.0)$ & & \\
\hline \multicolumn{6}{|l|}{ How frequent do you practice sex? } \\
\hline Once a while & $56(70.0)$ & $45(58.4)$ & $101(64.3)$ & 9.458 & 0.009 \\
\hline Always & $24(30.1)$ & $32(41.6)$ & $56(35.7)$ & & \\
\hline Total & $80(100.0)$ & $77(100.0)$ & $157(100.0)$ & & \\
\hline \multicolumn{6}{|c|}{ Does practicing safe sex protect you from Hepatitis B infection? } \\
\hline Yes & $131(59.8)$ & $117(49.2)$ & $248(54.3)$ & 5.220 & 0.022 \\
\hline No & $88(40.2)$ & $121(50.8)$ & $209(45.7)$ & & \\
\hline Total & $219(100.0)$ & $238(100.0)$ & $457(100.0)$ & & \\
\hline \multicolumn{6}{|l|}{ Have you practicing abstinence? } \\
\hline Yes & $106(48.4)$ & $218(82.9)$ & $324(67.2)$ & 64.503 & 0.001 \\
\hline No & $113(51.6)$ & $45(17.1)$ & $158(32.8)$ & & \\
\hline Total & $219(100.0)$ & 263(100.0) & $482(100.0)$ & & \\
\hline \multicolumn{6}{|c|}{ Do you believe that abstinence can be practiced by youth? } \\
\hline Yes & 198(85.3) & $3(27.3)$ & 201(82.7) & 130.915 & 0.001 \\
\hline No & $34(14.7)$ & $8(72.7)$ & $42(17.3)$ & & \\
\hline Total & 232(100.0) & $11(100.0)$ & $243(100.0)$ & & \\
\hline
\end{tabular}

Table 9: Distribution of respondents on beliefs (post -intervention) regarding personal safe sex practice and abstinence. 


\begin{tabular}{|l|c|c|c|c|}
\hline Do you always use condom? & & Group & $\boldsymbol{\chi}^{\mathbf{2}}$ & P-value \\
\hline & Control & Intervention & & \\
\hline Yes & $76(25.3)$ & $159(53.0)$ & 48.189 & 0.001 \\
\hline No & $224(74.7)$ & $141(47.0)$ & & \\
\hline Total & $300(100.0)$ & $300(100.0)$ & & \\
\hline
\end{tabular}

Table 10: Testing Null hypothesis 2 using $\chi^{2}$ test in the use of condoms between intervention and control groups.

Table 9 to which chi-square test was applied showed a significant number of respondents had sex $(\chi 2=13.83 ; \mathrm{p}=0.001)$ and practice was 'once in a while' $(\chi 2=9.46 ; \mathrm{p}=0.008)$. In addition, a significant number practiced abstinence $(\chi 2=64.5 ; \mathrm{p}=0.001)$ and believed abstinence could be practiced by youth $(\chi 2=130.92$; $\mathrm{p}=0.001$ ). The second hypothesis (Table 10 ) showed a significant difference in the use of condoms $(\chi 2=48.19 ; \mathrm{p}=0.001)$ post intervention between intervention and control group using chi-square test.

Studies show that adolescents who believe their peers are using condoms are also more than twice as likely to use condoms compared to teens who do not believe their peers use condoms (Diclemente,1991). A study done in Ethiopia on youth revealed that only 58.5\% used condom [11] and according to the Health Impact Evaluation conducted in Ethiopia in 2008 by Global Fund, among women aged 15-24 years, those who reported consistent condom use were $48 \%$, and abstinence from sex as means of preventing HIV infection was $58 \%$.

For a successful peer education country program on HBV prevention interventions for youth/adolescents, more research needs to be done in different parts of the country to get more substantial evidence regarding the models and education interventions that will be effective and successful for different age groups and different socio-cultural contexts.

\section{Conclusion}

The effects of a peer education intervention in schools are numerous despite a short follow-up period: increase in the level of knowledge, interest to have a hepatitis B test done, and the use of condoms among adolescents. Implementing secondary school targeted peer education interventions for disease prevention and control of HBV among school youth by allocating appropriate amounts of resources (money, man power, materials and time) by the government authorities is essential. State government needs to encourage heads of secondary schools to change their attitudes for learning to incorporate short messages for HBV prevention into weekly school activities like during school assembly, PTA meetings etc. Advocating to the state government for a coordination committee under the unit of disease control to coordinate solely viral hepatitis activities through a multi-sectoral approach. The state government needs to plan to have regular fora to collaborate with civil society groups, donor community and other stakeholders to implement hepatitis B prevention and control programs. Public enlightenment through media platforms need to occur to raise awareness on HBV infection (routes of transmission, different prevention methods available including importance of screening for HBV and vaccination).

A significant number of adolescent respondents had sex and practiced abstinence and believed abstinence could be practiced by youth. This is important in changing attitudes and behavior. Peer education interventions need to also target schoolteachers for an increase in level of knowledge so as to influence students on HBV and provide effective supervision.

\section{Bibliography}

1. World Health Organization. Hepatitis B. WHO updates (2017).

2. Spearman CW., et al. "Hepatitis B in sub-Saharan Africa: strategies to achieve the 2030 elimination targets". The Lancet Gastroenterology and Hepatology 2 (2017): 900-909.

3. National AIDS/STIs Control Program. Introduction. Guidelines for the Prevention, Treatment and Care of Viral Hepatitis in Nigeria. Federal Ministry of Health (2016): 1-30.

4. Okonkwo UC., et al. "Knowledge of hepatitis B virus infection among traders". Nigeria Journal of Clinical Practice 20 (2017): 415-420.

5. AIDS Info. HIV/AIDS related opportunistic infections and coinfections (2016).

6. Ikobah J., et al. "The prevalence of hepatitis B virus infection in Nigerian children prior to vaccine introduction into the National Programme on Immunization schedule". Pan African Medical Journal 23 (2016).

7. Federal Ministry of Health. National Training Manual on Peerto-Peer Youth health education. Growing girls and women in Nigeria (G-Win Project). (2013): 1-142.

8. Chingle MP., et al. "Risk perception of hepatitis B infection and uptake of hepatitis B vaccine among students of tertiary institution in Jos". Annals of African Medicine 16 (2017): 59-64.

9. Tung CS and Middleman A B. "An evaluation of school-level factors used in a successful school-based hepatitis B immunization initiative". Journal of Adolescent Health 37 (2005): 61-68. 
10. Takele M., et al. "Effects of peer education intervention on HIV/AIDS related sexual behaviors of secondary school students in Addis Ababa, Ethiopia: a quasi-experimental study (2015).

11. USAID/Ethiopia. HIV/AIDS profile. Addis Ababa, Ethiopia: USAID-Ethiopia Country Office (2010).

Volume 3 Issue 11 November 2019

(C) All rights are reserved by Mary Mathew., et al. 